\title{
Freqüência de diabetes mellitus e hiperglicemia em mulheres chagásicas e não-chagásicas
}

\author{
Hyperglycemia and diabetes mellitus in chagasic \\ and non-chagasic women
}

\author{
Vitorino Modesto dos Santos, Selma Freire de Carvalho da Cunha, Vicente de Paula \\ Antunes Teixeira, Jaqueline Pontes Monteiro, Jenner Arruda Modesto dos Santos, \\ Taciana Arruda Modesto dos Santos, Lister Arruda Modesto dos Santos \\ e Daniel Ferreira da Cunha
}

\begin{abstract}
Resumo Estudo retrospectivo de 647 mulheres com idade ž 40 anos, atendidas no HospitalEscola da FMTM, Uberaba-MG. As três sorologias para a doença de Chagas foram negativas nas controles ( $n=285)$ e positivas nas chagásicas $(n=362)$, que foram classificadas nas formas indeterminada $(n=125)$, megas $(n=58)$ e cardíaca $(n=179)$. Diabetes mellitus foi definido por duas glicemias em jejum • $140 \mathrm{mg} / \mathrm{dl}$ e hiperglicemia por glicemia em jejum $>110 \mathrm{mg} / \mathrm{dl}$. Os grupos foram comparados pelos testes do c2, análise de variância, " $t$ " de Student, Kruskal-Wallis e Mann-Whitney, considerando-se significativo $p<0,05$. chagásicas e controles estavam pareadas quanto à idade, o índice de massa corporal e a cor. Diabetes mellitus foi mais freqüente na forma cardíaca (15,1\%), comparada com as controles $(7,4 \%)$, megas $(7,4 \%)$ e assintomáticas $(5,6 \%)$, o mesmo ocorrendo com a hiperglicemia $(37,4 \%, 26,7 \%, 25,9 \%$ e 27,2\%, respectivamente), achados que estão de acordo com possível desnervação parassimpática causada pelo Trypanosoma cruzi e conseqüente predomínio da atividade simpática.
\end{abstract}

Palavras-chaves: Doença de Chagas. Disfunção autonômica. Hiperglicemia. Diabetes mellitus.

\begin{abstract}
Medical records of 440 years old female seen at University Hospital from June/93 to July/95 were submitted to a cross-sectional study. According to Chagas' disease tests, patients were divided into chagasic $(n=362)$ and controls $(n=285)$. Diabetes mellitus was defined on the basis of two fasting blood glucose levels $\cdot 140 \mathrm{mg} / \mathrm{dl}$ and hyperglycemia as fasting blood glucose $>110 \mathrm{mg} / \mathrm{dl}$. Chagasic patients were divided into groups with the cardiac form of the disease $(n=179)$, with megas $(n=58)$, and asymptomatic $(n=125)$. Groups were compared by the $c^{2}$ test, analysis of variance, Student's " $t$ " test, and Kruskal-Wallis and Mann-Whitney tests. A significant difference was assumed when $p<0.05$. Chagasic and control groups were matched for age, white color and body mass index. Diabetes mellitus was more prevalent in patients with the cardiac form of Chagas' disease than in controls, or patients with the megas or the asymptomatic form $(15.1 \%$, $7.4 \%, 7.4 \%$, and $5.6 \%$, respectively); the same was observed for hyperglycemia $(37.4 \%, 26.7 \%$, $25.9 \%, 27.2 \%)$, in agreement with the hypothesis that the reduced parasympathetic activity caused by Trypanosoma cruzi leads to relative sympathetic hyperactivity.
\end{abstract}

Key-words: Chagas' disease. Autonomic dysfunction. Hyperglycemia. Diabetes mellitus.

\footnotetext{
Departamento de Clínica Médica e Curso de Pós-graduação em Patologia da Faculdade de Medicina do Triângulo Mineiro, Uberaba MG.

Apoio financeiro: Fundação de Ensino e Pesquisa de Uberaba (FUNEPU).

Endereço para correspondência: Prof. Daniel Ferreira da Cunha. Disciplina de Nutrologia do Hospital Escola da FMTM. Av. Getúlio Guaritá s/nº, 38025-440 Uberaba, MG, Brasil. Tel: 5534 318-5335. Fax: 5534 312-6640.

E-mail: dfcnutro@mednet.com.br

Recebido para publicação em 7/8/98
} 
$\mathrm{Na}$ fase aguda da doença de Chagas humana ocorre parasitemia, que usualmente acomete o componente parassimpático do sistema nervoso do coração e do aparelho digestivo 323639 . A doença geralmente evolui para a fase crônica, manifestando-se com miocardiopatia e síndromes digestivas associadas aos megas ${ }^{32}$, com hemoculturas e xenodiagnósticos positivos ${ }^{20}{ }^{21} \mathrm{e}$ a presença de formas amastigotas do Trypanosoma cruzi na veia central da supra-renal ${ }^{4446}$.

O pâncreas pode ser afetado na doença de Chagas $^{312}{ }^{26}$. Na fase aguda, o T. cruzi promove reação inflamatória pancreática ${ }^{2834}$, que na fase crônica poderia evoluir para fibrose, calcificação e atrofia do parênquima ${ }^{48}$, fenômenos eventualmente associados com alterações funcionais exócrinas e endócrinas ${ }^{11}{ }^{32}$. Em chagásicos crônicos necropsiados, também foram descritas hiperplasia das ilhotas ${ }^{45}$ e inflamação com destruição de gânglios parassimpáticos intrapancreáticos ${ }^{37}$.

Estudos em animais indicam que a desnervação parassimpática afeta a secreção pancreática ${ }^{9}$. A possibilidade de associação da doença de Chagas com hipoinsulinismo e hiperglicemia ${ }^{35}$ foi sugerida em 1960 e a determinação das curvas glicêmica e insulínica em chagásicos assintomáticos mostrou menor resposta insulínica à sobrecarga oral e venosa de glicose ${ }^{1429}$. Dessa forma, é possível que muitos chagásicos crônicos apresentem desnervação parassimpática e alteração de células beta pancreáticas, processos que explicariam anormalidades ocasionalmente observadas nas curvas insulínicas e glicêmicas.

A hipótese do presente estudo é que eventuais alterações pancreáticas acarretariam menor reserva insulínica nos indivíduos com infecção crônica pelo T. cruzi, fenômeno que se manifestaria clinicamente por hiperglicemia ou diabetes mellitus (DM). O objetivo do presente estudo foi comparar a freqüência de hiperglicemia e de DM entre mulheres chagásicas e controles com idade $\cdot 40$ anos.

\section{MATERIAL E MÉTODOS}

O presente trabalho foi realizado na Faculdade de Medicina do Triângulo Mineiro (FMTM) em Uberaba, MG, após aprovação da Comissão de Ética em Pesquisa da Instituição. Os nomes e registros das pacientes foram obtidos de um arquivo eletrônico extraído do Computador Central do Departamento de Sistema de Informática e Métodos e do Instituto de Desenvolvimento Tecnológico do Hospital Escola da FMTM (HE-FMTM). Os dados foram organizados em banco de dados eletrônico planejado de forma a incluir glicemias em jejum e três exames sorológicos para doença de Chagas (imunofluorescência, hemaglutinação e fixação do complemento) realizados em pacientes adultos atendidos no período entre junho de 1993 e julho de 1995. No banco de dados original estavam registrados os testes para doença de Chagas, a glicemia em jejum (glicemia 1) realizada em concomitância com os testes sorológicos além de informações gerais sobre os pacientes, incluindo o nome, o registro no Serviço de Arquivo Médico e Estatística (SAME), a idade, o sexo, a cor, a procedência e o endereço.

As informações clínicas foram obtidas em prontuários arquivados no SAME. A coleta e registro de informações foram realizados por um único observador para homogeneidade nos critérios diagnósticos. O tamanho da amostra a ser estudada foi calculado utilizando-se a prevalência média esperada de DM de $8 \%$, com faixa de variação entre 6 e 10\% e margem de erro de $5 \%$.

Para assegurar a aleatoriedade na escolha da amostra, os números dos registros inicialmente obtidos $(n=3.854)$ foram dispostos em ordem crescente. Dos primeiros 800 prontuários de pacientes do sexo feminino, foram selecionados todos os casos de pacientes com idade igual ou superior a quarenta anos. A escolha desse limite de idade decorreu da maior probabilidade de alterações da glicemia e maior prevalência de DM em mulheres idosas e de meia-idade ${ }^{81024}$.

Os seguintes critérios de exclusão de casos foram adotados: 1) pacientes com somente uma ou duas das três sorologias positivas para doença de Chagas; 2) pacientes com formas mistas da doença de Chagas (cardíaca mais megas); 3) casos em que a análise do prontuário médico confirmasse ou permitisse inferir eventual concomitância de infusão intravenosa de soro glicosado com a coleta de sangue para medida da glicemia; 4) diagnósticos de hipertiroidismo, alcoolismo, síndrome pós-gastrectomia, gravidez ou uso de corticosteróides; 5) estado clínico compatível com síndrome da resposta de fase aguda (RFA), suspeita ou definida. A RFA foi diagnosticada pela ocorrência recente (período 
$<10$ dias) de infecção, trauma, acidente vascular encefálico ou pós-operatório imediato; situações em que pode haver hiperglicemia em conseqüência das alterações metabólicas associadas ao estresse 74950 .

Além do registro dos dados clínicos e dos medicamentos de uso regular, procurou-se documentar o peso e a altura das pacientes, para cálculo do índice de massa corporal (IMC) e o resultado de uma segunda glicemia em jejum (glicemia 2), realizada na data mais próxima à do exame sorológico para doença de Chagas (interstício máximo de dois meses). As pacientes foram classificadas como brancas e não-brancas, de acordo com a cor registrada no prontuário médico. Foram consideradas não-brancas as descritas como negras, pardas ou mulatas.

As 647 pacientes da amostra foram classificadas em chagásicas $(n=362)$ e não-chagásicas ou controles $(n=285)$, com base na concomitância de positividade das três reações sorológicas para a doença de Chagas. Foram consideradas como controles as pacientes que apresentaram as três sorologias negativas para a doença de Chagas. Para melhor caracterização clínica, o grupo das pacientes chagásicas foi subdividido ${ }^{32}{ }^{36}$ nas formas: 1) megas, definida pela presença de megaesôfago: diagnosticado à radioscopia e à radiografia contrastada do esôfago e megacólon, definido radiologicamente ou com base na história clínica, mediante comprovação de ectasia do orgão no enema opaco ou pela presença de constipação intestinal crônica superior a dez dias; 2) cardíaca, definida pela presença de alterações eletrocardiográficas (extra-sístoles ventriculares; bloqueios atrioventriculares e do ramo direito do feixe de His, associado ou não com bloqueio divisional ântero-superior esquerdo e fibrilação atrial) ${ }^{33}$ ou radiológicas, verificadas por evidências de cardiomegalia; 3) assintomática, nos casos de positividade das três reações sorológicas para doença de Chagas na ausência de sintomas e sinais classicamente atribuídos à doença.

O diagnóstico de DM foi estabelecido ${ }^{10} 19$ por um dos seguintes modos: 1) registro de pelo menos duas glicemias em jejum superiores a $140 \mathrm{mg} / \mathrm{dl}$; 2) diagnóstico prévio, firmado por especialista. A hiperglicemia foi definida pelo registro de glicemia em jejum acima de $110 \mathrm{mg} / \mathrm{dl}$.

Foi possível avaliar o estado nutricional de 182 pacientes $(28,1 \%$ da amostra) através de dados de peso e altura registrados nos prontuários médicos. O índice de massa corporal (IMC) foi calculado pela seguinte expressão: IMC = peso $(\mathrm{kg}) /$ altura $\left(\mathrm{m}^{2}\right)$. De acordo com o IMC as pacientes foram classificadas ${ }^{15} \mathrm{em}$ : 1) subnutridas: IMC $<18,5 \mathrm{~kg} / \mathrm{m}^{2} ; 2$ ) normais: IMC entre 18,5 e $27 \mathrm{~kg} / \mathrm{m}^{2}$; 3 ) obesas: IMC > $27 \mathrm{~kg} / \mathrm{m}^{2}$.

Os resultados foram considerados significativos ${ }^{27}$ quando a probabilidade de rejeição da hipótese de nulidade foi menor que $5 \%(p<0,05)$. As variáveis contínuas com distribuição normal foram expressas em termos de média \pm desvio padrão. O teste t não-pareado de Student foi utilizado na comparação entre dois grupos. A análise de variância foi empregada na comparação entre mais de dois grupos, eventualmente após correção de Bonferroni. As variáveis contínuas sem distribuição normal foram expressas em mediana e valores mínimos e máximos; neste caso o teste de Wilcoxon Mann-Whitney foi empregado na comparação entre dois grupos. O teste de Kruskal-Wallis foi aplicado na comparação entre mais de dois grupos. As proporções foram comparadas por meio dos testes do $\mathrm{c}^{2}$ (qui-quadrado) ou do exato de Fisher. Para melhor estudo comparativo das características gerais entre os grupos chagásicas e controle, aplicou-se a Correlação de Pearson para: idade vs IMC, IMC vs glicemia e idade $v s$ glicemia. Para essas duas últimas variáveis, a correlação foi também avaliada de modo específico para cada uma das formas clínicas da doença de Chagas. Para afastar a possibilidade de que níveis glicêmicos muito elevados pudessem estar associados a eventual descompensação diabética e não a fatores como IMC ou idade, a correlação de Pearson foi aplicada apenas aos casos com glicemia abaixo de $200 \mathrm{mg} / \mathrm{dl}$.

\section{RESULTADOS}

Foram estudados 647 prontuários de pacientes do sexo feminino, contendo informações suficientes para análise. Os grupos chagásicas $(n=362)$ e controles $(n=285)$ não diferiram quanto à idade, à porcentagem de mulheres de cor branca, ao IMC e às glicemias (Tabela 1). O IMC foi calculado para 182 mulheres $(28,1 \%)$ em que se pode obter dados confiáveis de peso e altura. Chagásicas e controles apresentaram IMC médios estatisticamente similares, fato que também pode ser observado quando as pacientes foram classificadas em normais, obesas e subnutridas (Figura 1). 
Tabela 1 - Características gerais e metabólicas de chagásicas e controles, registradas em 647 mulheres com idade • 40 anos, atendidas no HE-FMTM.

\begin{tabular}{lcc}
\hline Parâmetros & Chagásicas $(\mathrm{n}=362)$ & Controles $(\mathrm{n}=285)$ \\
\hline Idade (anos) & $57,3 \pm 10,4$ & $57,0 \pm 11,3$ \\
Cor (branca, em \%) & 77,1 & 75,8 \\
Índice de massa corporal $\left(\mathrm{kg} / \mathrm{m}^{2}\right)$ & $26,8 \pm 5,8$ & $26,2 \pm 5,6$ \\
Glicemia 1 $(\mathrm{mg} / \mathrm{dl})$ & $96(52-448)$ & $95(60-363)$ \\
Glicemia 2 $(\mathrm{mg} / \mathrm{dl})$ & $100(60-352)$ & $98,5(70-476)$ \\
Hiperglicemia (\%) & 32,0 & 26,7 \\
Diabetes mellitus (\%) & 10,5 & 7,4
\end{tabular}

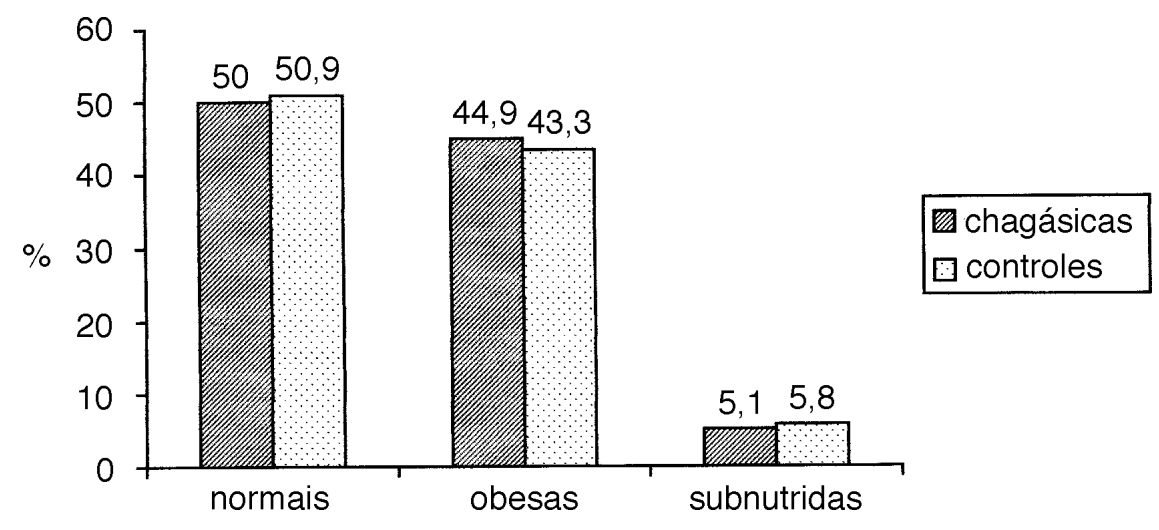

Figura 1 - Estado nutricional de mulheres chagásicas e controles com idade 440 anos, atendidas no HE-FMTM entre junho de 1993 e julho de 1995.

A freqüência de DM na amostra estudada foi de $9,1 \%$. Chagásicas e controles apresentaram freqüência similar de DM, respectivamente 10,5 e 7,4\%, o mesmo se verificando quanto à freqüência de hiperglicemia e níveis de glicemia em jejum (Tabela 1). A subdivisão das pacientes chagásicas nas formas clínicas principais identificou as formas cardíaca $(n=179)$, megas $(n=58)$ e assintomática $(n=125)$. O grupo com a forma megas teve média de idade significativamente superior às médias de idade das demais formas clínicas e à do grupo controle. Não houve diferença estatística na porcentagem de pacientes de cor branca, nem nos valores do IMC e da glicemia 2. No entanto, a glicemia 1 e as freqüências de hiperglicemia e de DM foram estatisticamente maiores nas chagásicas com a forma cardíaca (Tabela 2).

Tabela 2 - Características gerais e metabólicas do grupo controle e das formas cardíaca, megas e assintomática, registradas em 647 mulheres com idade • 40 anos, atendidas no HE-FMTM.

\begin{tabular}{lcccc}
\hline Parâmetros & $\begin{array}{c}\text { Controle } \\
(\mathrm{n}=285)\end{array}$ & $\begin{array}{c}\text { Cardíaca } \\
(\mathrm{n}=178)\end{array}$ & $\begin{array}{c}\text { Megas } \\
(\mathrm{n}=58)\end{array}$ & $\begin{array}{c}\text { Assintomática } \\
(\mathrm{n}=125)\end{array}$ \\
\hline Idade (anos) (a) & $57,0 \pm 11,3$ & $57,8 \pm 10,5$ & $60,0 \pm 10,1$ & $55,4 \pm 10,5$ \\
Cor (branca, em \%) & 75,8 & 75,4 & 75,9 & 80,0 \\
IMC (kg/m²) & $26,2 \pm 5,6$ & $27,7 \pm 5,9$ & $23,1 \pm 4,3$ & $27,0 \pm 5,8$ \\
Glicemia 1 (mg/dl) ${ }^{(b)}$ & $95(60-363)$ & $100(62-445)$ & $94,5(69-309)$ & $95(52-100)$ \\
Glicemia 2 (mg/dl) & $98,5(70-476)$ & $101(60-346)$ & $98(61-338)$ & $94(71-352)$ \\
Hiperglicemia (\%) (b) & 26,7 & 37,4 & 25,9 & 27,2 \\
DM (\%) ${ }^{(\text {b) }}$ & 7,4 & 15,1 & 7,4 & 5,6 \\
\hline
\end{tabular}

IMC = índice de massa corporal. $\mathrm{DM}=$ diabetes mellitus. ${ }^{\left({ }^{a}\right)}$ Grupo com a forma megas difere dos demais. ${ }^{(b)}$ Grupo com a forma cardíaca difere dos demais. 
Por ordem de freqüência, os sintomas e diagnósticos relacionados com a função cardíaca registrados nos 647 casos analisados foram: palpitação $12,5 \%(n=78)$, dispnéia $10,5 \%$ $(n=68)$, dor precordial $9,4 \%(n=61)$ e insuficiência cardíaca 3,9\% $(n=25)$. Exceto pela insuficiência cardíaca, chagásicas e controles diferiram estatisticamente quanto à freqüência de sintomas associados à descompensação cardíaca, incluindo dispnéia $(14,4 \%$ vs $5,6 \%)$, dor precordial (12,7\% vs 5,3\%) e palpitação (16,8\% vs $6,0 \%$ ) (Tabela 3 ). A ocorrência de HAS na

Tabela 3 - Freqüência (\%) de sintomas e diagnósticos mais comuns, registrados em 647 mulheres chagásicas e controles com idade $\cdot 40$ anos, atendidas no HE-FMTM.

\begin{tabular}{lcc}
\hline Parâmetros $\left(n^{\circ}\right.$ de casos) & Chagásicas $(n=362)$ & Controles $(n=285)$ \\
\hline Dispnéia $(n=68)^{*}$ & 14,4 & 5,6 \\
Dor precordial $(n=61)^{*}$ & 12,7 & 5,3 \\
Palpitação $(n=78)^{*}$ & 16,8 & 6,0 \\
ICC $(n=25)$ & 3,6 & 4,2 \\
HAS $(n=248)$ & 37,8 & 38,9 \\
\hline
\end{tabular}

ICC = insuficiência cardíaca congestiva. HAS = hipertensão arterial sistêmica. ${ }^{*} p<0,05$.

forma cardíaca diferiu dos outros grupos, não havendo diferença estatística entre o grupo controle e as formas megas e assintomática. A freqüência desses sintomas e diagnósticos nas pacientes do grupo controle e nas formas clínicas da doença de Chagas é apresentada na Tabela 4.

Tabela 4 - Freqüência (\%) de sintomas e diagnósticos mais comuns do grupo controle e das formas cardíaca, megas e assintomática, registrados em 647 mulheres com idade $\cdot 40$ anos, atendidas no HE-FMTM.

\begin{tabular}{lcccc}
\hline $\begin{array}{l}\text { Parâmetros } \\
\left(\mathrm{n}^{\circ} \text { de casos) }\right.\end{array}$ & $\begin{array}{c}\text { Controle } \\
(\mathrm{n}=285)\end{array}$ & $\begin{array}{c}\text { Cardíaca } \\
(\mathrm{n}=178)\end{array}$ & $\begin{array}{c}\text { Megas } \\
(\mathrm{n}=58)\end{array}$ & $\begin{array}{c}\text { Assintomática } \\
(\mathrm{n}=125)\end{array}$ \\
\hline Dispnéia $(\mathrm{n}=68)^{(\mathrm{a})}$ & 5,6 & 18,4 & 5,2 & 12,8 \\
${\text { Dor precordial }(\mathrm{n}=61)^{(\mathrm{b})}}^{{ }^{(b)}}$ & 5,3 & 16,8 & 8,6 & 8,8 \\
Palpitação $(\mathrm{n}=78)^{\left({ }^{(c)}\right.}$ & 6,0 & 19,6 & 19,0 & 12,0 \\
ICC $(\mathrm{n}=25)^{(\mathrm{d})}$ & 4,2 & 5,6 & 5,2 & 0,0 \\
HAS $(\mathrm{n}=248)^{(b)}$ & 38,9 & 49,7 & 25,9 & 26,4 \\
\hline
\end{tabular}

ICC = insuficiência cardíaca congestiva. HAS = hipertensão arterial sistêmica. ${ }^{(a)}$ Grupo com a forma megas difere da forma cardíaca. ${ }^{(b)}$ Grupo com a forma cardíaca difere dos demais. (c) Grupo controle difere dos demais. ${ }^{(\mathrm{d})}$ Grupo com a forma assintomática difere dos demais.

\section{DISCUSSÃO}

Neste estudo, documentou-se maior freqüência de diabetes mellitus e de hiperglicemia em mulheres chagásicas, quando comparadas ao grupo controle. Esta conclusão só foi possível após a categorização das chagásicas nas formas clínicas (Tabela 2), permitindo a constatação da maior porcentagem de casos de DM entre as chagásicas com a forma cardíaca $(15,1 \%)$. Esses resultados não podem ser atribuídos a eventual diferença de idade entre os grupos ${ }^{40}$, já que ocorreu o planejado pareamento com relação a essa variável, como demonstram as médias de idade dos dois grupos: chagásicas $(57,3 \pm 10,4$ anos) e controles (57,0 $\pm 11,3$ anos). Da mesma forma, não houve diferença na proporção de mulheres brancas vs não-brancas entre os grupos, pareamento importante, considerando- se que fatores raciais poderiam influir na evolução e na forma de apresentação clínica da doença de Chagas $^{30}$. O pareamento do estado nutricional, verificado entre chagásicas e controles, sugere que o fator adiposidade não interferiu na maior freqüência de hiperglicemia e DM entre os grupos $^{31}$.

Nas mulheres com a forma cardíaca, registrouse correlação positiva e significativa entre os níveis de glicemia e a idade (Figura 2), o que sugere que os chagásicos com maiores desnervações parassimpáticas manifestem a exaustão do sistema regulador da normoglicemia mais precocemente que os indivíduos nãochagásicos (ou não desnervados). A eventual presença do T. cruzi na veia central da supra- 


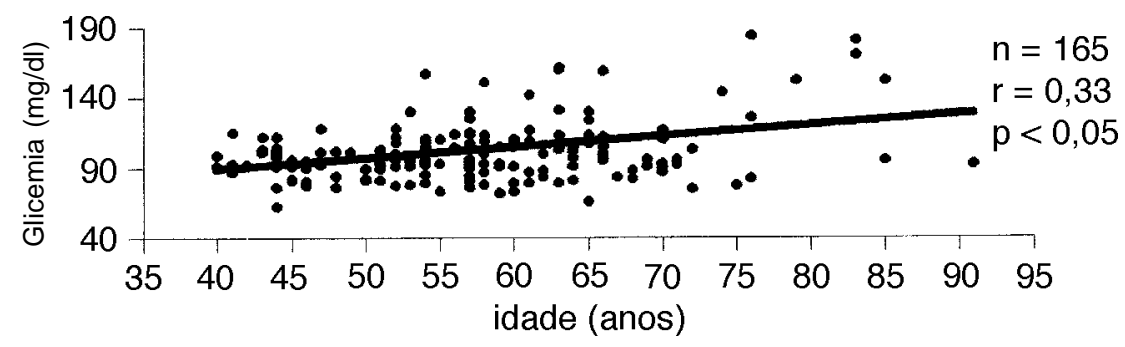

Figura 2 - Correlação entre idade e glicemia 1 em mulheres chagásicas com a forma cardíaca e idade 40 anos, atendidas no HE-FMTM entre junho de 1993 e julho de 1995.

renal ${ }^{46}$ também poderia estar associada a algum distúrbio funcional, incluindo alteração na produção de corticosteróides e catecolaminas, hormônios que têm influência nos níveis da glicemia ${ }^{17}$.

Além de eventuais alterações no eixo ênteroinsular, decorrentes da desnervação intra e extrapancreática ${ }^{1229}$, a hiperglicemia poderia decorrer de desequilíbrio autonômico associado à desnervação parassimpática ${ }^{23} 42$. $O$ aumento dos níveis circulantes ou da atividade tecidual de catecolaminas elevaria a taxa de glicogenólise ${ }^{17}$ e promoveria maior resistência periférica à insulina, fenômenos que se manifestariam por níveis de glicemia mais elevados. O predomínio do tônus simpático tem sido relatado em outras cardiopatias ${ }^{4}$, podendo explicar as arritmias supraventriculares ou ventriculares ${ }^{25}$, a maior dispersão da atividade elétrica miocárdia ${ }^{43}$ e a morte súbita ${ }^{18}$. Também não se pode excluir que a hiperglicemia decorra da secreção de interleucinas associada com a miocardite chagásica em atividade ${ }^{6} 164146$.

No presente estudo, a HAS foi comum em controles e chagásicas, sendo mais freqüente entre as chagásicas com a forma cardíaca, o que está de acordo com o observado por Guariento ${ }^{13}$. A maior freqüência de palpitação, dispnéia e precordialgia nas mulheres chagásicas, em especial as com a forma cardíaca está dentro do esperado e de acordo com a patogenia dessa doença, que acomete a inervação parassimpática do coração ${ }^{5}$, a função miocárdica e a microcirculação coronariana $^{22} 383947$.

Uma das limitações deste estudo foi a não documentação da glicemia 2 em alguns casos. Entretanto, a freqüência de hiperglicemia, baseada na glicemia 1 , foi maior nas chagásicas da forma cardíaca, o que indica a possibilidade de que o diagnóstico de DM possa ter sido subestimado. Outra possível falha seria a documentação do estado nutricional de apenas 1/3 da amostra, fato de menor importância se considerarmos a distribuição homogênea de eventuais erros na obtenção dos dados amostrais. Estudos prospectivos que incluam a análise espectral da atividade elétrica do coração e a determinação dos níveis séricos da hemoglobina glicosilada e do peptídeo $\mathrm{C}$ poderiam confirmar essa maior freqüência de distúrbios da glicemia na forma cardíaca da doença de Chagas.

Concluindo, no presente estudo se documentou que pacientes chagásicas com a forma cardíaca crônica, apresentaram maior freqüência de hiperglicemia e de DM que os outros grupos. Possíveis explicações para esse achado incluem: 1) atividade simpática excessiva (absoluta ou relativa ao grau de desnervação parassimpática) nas chagásicas com a forma cardíaca; e 2) hipoinsulinemia (relativa ou absoluta) decorrente do comprometimento funcional das células beta pancreáticas, fenômeno associado à infecção pelo T. cruzi.

\section{REFERÊNCIAS BIBLIOGRÁFICAS}

1. Amorim DS, Marin Neto JA. Functional alterations of the autonomic nervous system in Chagas' heart disease. Revista Paulista de Medicina 113:772-784, 1995.

2. Barbosa AJA. Sistema APUD e doença de Chagas. Revista da Sociedade Brasileira de Medicina Tropical 25(supl):59-61, 1992.
3. Britto-Costa R, Neto Júnior EM, Mabtum J. Estudo sobre a secreção glandular exócrina do aparelho digestivo e dos brônquios na moléstia de Chagas humana. Revista do Instituto de Medicina Tropical de São Paulo 15:227234, 1973.

4. Chakko S, Mulingtapang RF, Huikuri HV, Kessler KM, Materson BJ, Meyerburg RJ. Alterations in heart rate 
variability and its circadian rhythm in hypertensive patients with left ventricular hypertrophy free of coronary artery disease. American Heart Journal 126:1364-1372, 1993.

5. Chapadeiro E, Florencio RFC, Afonso PC, Beraldo PS, Jesus PC, Junqueira LF. Neuronal counting and parasympathetic dysfunction in the hearts of chronically Trypanosoma cruzi infected rats. Revista do Instituto de Medicina Tropical de São Paulo 33:337-341, 1991.

6. Clark IA, AI Yaman FM, Jacobson LS. The biological basis of malarial disease. International Journal for Parasitology 27:1237-1249, 1997.

7. Cunha DF, Vieira CO, Silva GP, Erédia GR, Teixeira VPA. Reação de fase aguda e parasitismo na veia central da supra-renal de chagásicos crônicos. Revista da Sociedade Brasileira de Medicina Tropical 27:128, 1994.

8. Cunha SFC, Oliveira JED. Dieta e saúde na América Latina e Caribe: informe correspondente ao Brasil. Ribeirão Preto: Fundação Simpósio Brasileiro de Alimentação e Nutrição, 1993.

9. Frohman LA, Ezdinli EZ, Javid R. Effect of vagal stimulation on insulin secretion. Diabetes 16:443-448, 1967.

10. Genuth S. Classification and diagnosis of diabetes mellitus. Medical Clinics of North America 66:1191-1207, 1982.

11. Grendell JH, Cello JP. Chronic pancreatitis. In: Sleisenger $\mathrm{MH}$, Fordtran JS (eds) Gastrointestinal disease. Pathophysiology. Diagnosis. Management. $5^{\text {th }}$ edition. WB Saunders, Philadelphia. p. 1654-1681, 1993.

12. Guariento ME, Olga E, Muscelli A, Gontijo JAR. Chronotropic and blood pressure response to oral glucose load in Chagas' disease. Revista Paulista de Medicina 112: 602-606, 1994.

13. Guariento ME, Orosz JEB, Morano FG, Parma MF, Rocha $\mathrm{RH}$, Freitas AG. Associação entre doença de Chagas crônica e hipertensão arterial em um serviço de referência. Revista da Sociedade Brasileira de Medicina Tropical 27(supl):139-140, 1994.

14. Guariento ME, Saad MJA, Muscelli EOA, Gontijo JAR. Heterogenous insulin response to an oral glucose load by patients with the indeterminate clinical form of Chagas' disease. Brazilian Journal of Medical and Biological Research 26:491-495, 1993.

15. Heymsfield SB, Tigh A, Zi-mian W. Nutritional assessment by anthropometric and biochemical methods. In: Shils, ME, Olson JA, Shike M, (ed.) Modern Nutrition in Health and Disease. Lea and Febiger, Philadelphia. p. 812-841, 1994.

16. Higuchi ML. Chronic chagasic cardiopathy: the product of a turbulent host-parasite relationship. Revista do Instituto de Medicina Tropical de São Paulo 39: 53-60, 1997.

17. Hoffman BB, Lefkowitz RJ. Catecholamines, sympathomimetic drugs, and adrenergic receptor antagonists. In: Hardman JG, Gilman AG, Limbird LE, (eds) Goodman \& Gilman's pharmacological basis of therapeutics. $9^{\text {th }}$ edition. McGraw-Hill, New York. p. 199248, 1996.

18. Junqueira Júnior LF. Disfunção autonômica cardíaca, arritmogênese e morte súbita chagásica: perspectiva fisiopatológica. Revista da Sociedade Brasileira de Medicina Tropical 26(supl):30-35, 1993.

19. Karam JH, Forsham PH. Pancreatic hormones and diabetes mellitus. In: Greenspan FS, Baxter JD (eds) Basic and Clinical Endocrinology. $4^{\text {th }}$ edition. PrenticeHall International, New Jersey. p.571-634, 1994.

20. Levi GC, Kallás EG, Mendonça JS, Amato Neto V. Reaparecimento da positividade de xenodiagnóstico 15 anos após tratamento específico da doença de Chagas. Revista da Sociedade Brasileira de Medicina Tropical 27:179-180, 1994.

21. Luz ZMP, Coutinho MG, Cançado JR, Kretli AU. Alta positividade de hemoculturas repetidas em pacientes chagásicos crônicos não tratados. Revista da Sociedade Brasileira de Medicina Tropical 26 (supl):66-67, 1993.

22. Marin-Neto JA, Simões MV, Ayres-neto EM, Santos JLA, Gallo Júnior L, Amorim DS, Maciel BC. Studies of the coronary circulation in Chagas' heart disease. Revista Paulista de Medicina 113:826-834, 1995.

23. Matthews DR, Clark A. Neural control of the endocrine pancreas. Proceedings of the Nutrition Society 46:89-95, 1987.

24. Ministério da Saúde. Manual de Diabetes. 2. ${ }^{\text {a }}$ edição. Brasilia, 1993.

25. Moore EN. Mechanisms and models to predict a QTC effect. American Journal of Cardiology, New York 72:4B9B, 1993.

26. Neves MM, Patto RJ, Russo E, Borges DR. Insulin secretion in alcoholics with chagasic infection. Revista Brasileira de Patologia Clínica 23:127-130, 1987.

27. Noether GE. Introdução à estatística: uma abordagem não paramétrica. $2^{\mathrm{a}}$. ed. Rodrigues FW, Cordani LK (trad) Rio de Janeiro: Guanabara Dois, 1983.

28. Okumura M, Brito T, Silva LHP, Silva AC, Netto AC. The pathology of experimental Chagas' disease in mice: I. digestive tract changes, with a reference to necrotizing arteritis. Revista do Instituto de Medicina Tropical de São Paulo 2:17-28, 1960.

29. Oliveira LCM, Juliano Y, Novo NF, Neves MM. Blood glucose and insulin response to intravenous glucose by patients with chronic Chagas' disease and alcoholism. Brazilian Journal of Medical and Biological Research 26:1187-1190, 1993.

30. Piancastelli $\mathrm{CH}$, Rocha MOC, Gontijo ED, Guerra HL, Abreu CP, Neiva Júnior L, Antunes CMF. Associação entre sexo, cor e resultado do exame radiológico do esôfago em pacientes com sorologia positiva para $T$. cruzi, procedentes de bancos de sangue. Revista da Sociedade Brasileira de Medicina Tropical 26 (supl):119-120, 1993. 
31. Pi-Sunyer FX. Obesity. In: Shils ME, Olson JA, Shike M, (eds) Modern Nutrition in Health and Disease. Lea and Febiger, Philadelphia p. 984-1006, 1994.

32. Prata A. Chagas' disease. Infectious Diseases Clinics of North America 8:61-76, 1994.

33. Prata SP, Cunha DF, Cunha SFC, Prata SC, Nogueira N. Prevalência de alterações eletrocardiográficas em 2000 pacientes chagásicos idosos e não-idosos. Arquivos Brasileiros de Cardiologia 60:369-372, 1993.

34. Ramirez LEG. Susceptibilidade do hamster à infecção pelo Trypanosoma cruzi: estudos parasitológicos e histopatológicos comparativos com o camundongo. Tese para Professor de Titular. Faculdade de Medicina do Triângulo Mineiro. Uberaba, 1995.

35. Reis LCF, Oliveira HL, Vieira C. Curvas glicêmicas anormais observadas em pacientes com a forma crônica da moléstia de Chagas. Nota preliminar. Revista Goiana de Medicina 6:155-165, 1960.

36. Rezende JF. Aspectos da forma digestiva da doença de Chagas. Revista da Sociedade Brasileira de Medicina Tropical 27 (supl):73-74, 1994.

37. Rocha A, Oliveira LCM, Alves RS, Lopes ER. Despopulação neuronal pancreática em chagásicos crônicos. Revista da Sociedade Brasileira de Medicina Tropical 31:43-49, 1998.

38. Rossi MA. Microvascular changes as a cause of chronic cardiomyopathy in Chagas' disease. American Heart Journal 120:233-236, 1990.

39. Rossi MA, Ramos SG. Pathogenesis of chronic Chagas'disease. Cardiovascular Pathology 5:197-202, 1996.

40. Silva AC. Doença de Chagas no paciente idoso. Revista da Sociedade Brasileira de Medicina Tropical 25 (supl):69-70, 1992.

41. Silva JS, Vespa, GNR, Cardillo F, Cardoso MAG, Martins GA, Rodrigues V. O papel da citocinas no determinismo de resistência ou susceptibilidade à infecção pelo
Trypanosoma cruzi. Revista da Sociedade Brasileira de Medicina Tropical 26 (supl):17-18, 1993.

42. Smith PH, Madson KL. Interactions between autonomic nerves and endocrine cells of the gastroenteropancreatic system. Diabetologia 20 (supl):314-324, 1981.

43. Soares JD, Junqueira júnior LF. Incidência de arritmias associadas à manobra de Valsalva nas diversas formas clínicas da doença de Chagas. Revista da Sociedade Brasileira de Medicina Tropical 20 (supl):58, 1987.

44. Teixeira VPA. A supra-renal e a forma crônica da doença de Chagas. Revista da Sociedade Brasileira de Medicina Tropical 25 (supl):52-56, 1992.

45. Teixeira VPA, Almeida HO, Gobbi H, Barbosa AJA, Silveira SA, Reis MA, Araujo MBM. Avaliação morfométrica das ilhotas pancreáticas em chagásicos crônicos. Revista da Sociedade Brasileira de Medicina Tropical 22 (supl):80, 1989.

46. Teixeira VPA, Araújo MBM, Reis MA, Reis L, Silveira SA, Rodrigues MLP, Franquini Júnior J. Possible role of an adrenal parasite reservoir in the pathogenesis of chronic Trypanosoma cruzi myocarditis. Transactions of the Royal Society of Tropical Medicine and Hygiene 87:552554, 1993.

47. Torres FW, Acquatella H, Condado JA, Dinsmore R, Palacios IF. Coronary vascular reactivity is abnormal in patients with Chagas' heart disease. American Heart Journal 129:995-1001, 1995.

48. Vieira CB, Hadler WA. Estudo histológico da glândula parótida e do pâncreas no megaesôfago. Revista da Associação Médica Brasileira 7:89-96, 1961.

49. Wilmore DW. Pathophysiology of the hypermetabolic response to burn injury. Jounal of Trauma 30 (supl):4-6, 1990.

50. Wolfe RR, Klein S, Herndon DN, Jahhoor F. Substrate cycling in thermogenesis and amplification of net substrate flux in human volunteers and burned patients. Journal of Trauma, Baltimore 30 (supl):6-9, 1990. 